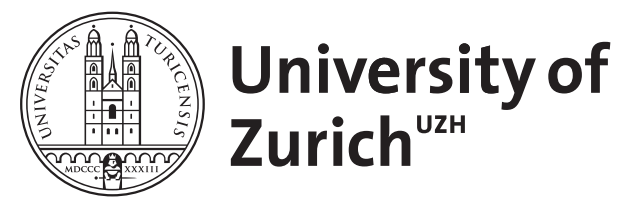

\title{
Functions of ABC transporters in plants
}

\author{
Kretzschmar, T ; Burla, B ; Lee, Y ; Martinoia, E ; Nagy, R
}

\begin{abstract}
ABC (ATP-binding cassette) proteins are ubiquitously found in prokaryotes and eukaryotes and generally serve as membrane-intrinsic primary active pumps. In higher plants, ABC proteins constitute a large family, grouped phylogenetically into eight clusters, subfamilies ABCA-ABCI (ABCH is not found in plants). ABC transporters shuttle substrates as diverse as lipids, phytohormones, carboxylates, heavy metals, chlorophyll catabolites and xenobiotic conjugates across a variety of biological membranes. To date, the largest proportions of characterized members have been localized to the plasma membrane and the tonoplast, with dominant implications in cellular secretion and vacuolar sequestration, but they are also found in mitochondrial, plastidal and peroxisomal membranes. Originally identified as tonoplastintrinsic proteins that shuttle xenobiotic conjugates from the cytosol into the vacuole, thus being an integral part of the detoxification machinery, $\mathrm{ABC}$ transporters are now recognized to participate in a multitude of physiological processes that allow the plant to adapt to changing environments and cope with biotic and abiotic stresses.
\end{abstract}

DOI: https://doi.org/10.1042/bse0500145

Posted at the Zurich Open Repository and Archive, University of Zurich ZORA URL: https://doi.org/10.5167/uzh-53838

Journal Article

Accepted Version

Originally published at:

Kretzschmar, T; Burla, B; Lee, Y; Martinoia, E; Nagy, R (2011). Functions of ABC transporters in plants. Essays in Biochemistry, 50(1):145-60.

DOI: https://doi.org/10.1042/bse0500145 
1 On the Functions of ABC Transporters in Plants

2

3 Tobias Kretzschmar ${ }^{1}$, Bo Burla ${ }^{1,2}$, Youngsook Lee ${ }^{2}$, Enrico Martinoia ${ }^{1,2}$, Réka

4 Nagy $^{1}$

5

$6{ }^{1}$ Institute of Plant Biology, University of Zurich, Zollikerstrasse 107, 8008 Zurich,

7 Switzerland

$8{ }^{2}$ POSTECH-UZH Global Research Laboratory, Division of Molecular Life

9 Sciences, POSTECH University of Science and Technology, Pohang, 790-784,

10 South Korea

12 Abstract

13 ATP Binding Cassette (ABC) proteins are ubiquitously found in prokaryotes and

14 eukaryotes and generally serve as membrane-intrinsic primary active pumps. In

15 higher plants, ABC proteins constitute a large family, phylogenetically structured

16 into eight clusters, namely the $A B C A$ to $A B C I$ subfamilies [1]. $A B C$ transporters

17 shuttle substrates as diverse as lipids, phytohormones, carboxylates, heavy

18 metals, chlorophyll catabolites and xenobiotic-conjugates across a variety of

19 biological membranes. To date, the largest proportions of characterized members

20 have been localized to the plasma membrane and the tonoplast with dominant

21 implications in cellular secretion and vacuolar sequestration, but they are also

22 found in mitochondrial, plastidal and peroxisomal membranes. Originally

23 identified as tonoplast-intrinsic proteins that shuttle xenobiotic-conjugates from 
24 the cytosol into the vacuole, thus being an integral part of the detoxification

25 machinery, $A B C$ transporters are now recognized to participate in a multitude of

26 physiological processes that allow the plant to adapt to changing environments

27 and cope with biotic and abiotic stresses.

\section{1. Introduction}

30 The plant $A B C$ protein family consists of full-size, half-size and several soluble

31 members that all share a cytosolic nucleotide-binding domain. Functional

32 transport units utilize ATP-hydrolysis to energize the transport of solutes across

33 membranes, independent of concentration gradients and membrane potentials.

34 Full-size members are organized in a modular fashion, consisting of two pore-

35 forming transmembrane domains alternating with two cytosolic nucleotide-binding

36 domains. Half-size members, which contain one transmembrane domain and one

37 nucleotide-binding domain, are thought to form dimers that act as functional units.

38 In most subfamilies, transmembrane domains precede nucleotide-binding

39 domains, a topology which is referred to as forward orientation, while the

40 opposite is considered a reverse orientation [2]. An additional N-terminal

41 transmembrane domain (TMD0) is characteristic to members of the ABCC

42 subfamily. In humans and yeast, the TMDO of some ABCC proteins was

43 demonstrated to be essential for correct protein targeting, without necessarily

44 having implications in substrate specificity and transport activity $[3,4]$. The plant

45 soluble $A B C$ proteins cluster in the $A B C E, A B C F$ and $A B C I$ subfamilies. However,

46 due to a primary focus on membrane-intrinsic half- and full-size proteins, they will 
47 not be addressed further in this review. Readers interested in this topic will find

48 information in two comprehensive reviews on plant $A B C$ proteins [2,5].

49 Genome wide inventories of $\mathrm{ABC}$ transporters in Arabidopsis, rice and poplar led

50 to the identification of more than 100 loci encoding for either half-size or full-size

51 transporters in each, making them nearly twice as numerous in plants as

52 reported for sequenced animal species. Half-size (WBC) and full-size (PDR)

53 members of the reverse oriented ABCG subfamily are abundantly found in plants.

54 No full-size ABCG transporters have been identified in animals. It is argued that

55 the expansion and diversification of the $A B C$ protein family in plants may be

56 tightly linked to their autotrophic and sessile lifestyle, requiring a highly plastic

57 development, interaction with the environment and maintenance of basic

58 metabolism under changing conditions.

59 As a secondary effect of nutrient acquisition from the soil, plants often take up

60 toxic heavy metals, which need to be secreted in order not to interfere with

61 metabolism. One possibility is reflux into the soil or secretion into the foliar

62 apoplast. Full-size ABCG members have been identified as candidates for

63 cadmium and possibly lead export [6,7]. Furthermore, ABC transporters play an

64 important role in the general detoxification mechanism. Extrinsic or intrinsic

65 potentially toxic compounds are first incorporated into conjugates, which are then

66 recognized by tonoplastic $A B C$ transporters and sequestered into the vacuole [8].

67 Phytohormones are major modulators of plant development and plant responses

68 to biotic and abiotic environmental stimuli. Sites of hormone production differ

69 from sites of hormone action and thus, export from producing cells, directed 
70 distribution within the plant body and specific uptake into receptive cells are

71 crucial for adequate plant function. $A B C$ transporters participate in the

72 translocation of the hormone auxin, a dominant developmental regulator [9], and

73 the hormone abscisic acid (ABA), implicated in abiotic stress responses $[10,11]$.

74 Plants possess an often species specific array of natural compounds that are

75 produced and deposited in a strictly regulated manner to directly interact with the

76 biotic environment such as deterring herbivores, warding off pathogens and

77 attracting symbionts or pollinators. Their biosynthetic pathways often involve

78 several organelles within a cell and different cell types within an organism.

79 Precursors as well as final products need to be transported across membranes

80 and in several cases ABC transporters are proposed as candidates. Best studied

81 in this respect is the abaxial epidermal deposition of antimicrobial compounds $82[12,13]$

84 2. Cellular Detoxification

85 First indications for ABC-mediated transport processes in plants were observed 86 when Martinoia et al. [14] began investigating how xenobiotics are detoxified in 87 plants. They found that potentially toxic compounds, bound to the tripeptide 88 glutathione through their thiol group (glutathione conjugates), are transported into 89 the vacuole independently from the proton motive force generated by two 90 vacuolar proton pumps. Initially, detoxification processes in plants and animals 91 are very similar, involving cytochrome P450 proteins and several transferases, 92 such as glutathione S-transferases, glucuronosyltransferases or 
93 glycosyltransferases [15]. Unlike animals which excrete modified, potentially toxic

94 compounds, plants internalize toxins in large central vacuoles. Two vacuolar

95 glutathione conjugate transporters, AtABCC1/AtMRP1 and AtABCC2/AtMRP2,

96 were identified [16]. Both proteins transport a broad range of glutathione

97 conjugates, glucuronates as well as a chlorophyll catabolite. Later studies have

98 shown that AtABCC1 is an efficient transporter of folates, which are known to be

99 stored in the vacuole and are required for methylation processes [17]. The latter

100 findings suggest that $A B C C$ s are not solely involved in cellular detoxification.

101 Proteomic data obtained for Arabidopsis vacuoles suggest that most ABCCs of

102 Arabidopsis reside in the tonoplast [18], and other ABCCs, additionally to

103 AtABCC1/2, may contribute to the overall glutathione conjugate transport activity.

104 Heavy metals are also stored within the central vacuole for detoxification

105 purposes and data suggest that phytochelatins and phytochelatin heavy metal

106 complexes are transported by ABCC-type transporters [19]. In contrast to $S$.

107 cerevisae, where an ABCC-type transporter, YCF1 (yeast cadmium factor 1),

108 catalyzes the transport of glutathion-cadmium complexes [20], and to S. pombe,

109 where Hmt1, a half-size $A B C$ transporter, sequesters phytochelatin [21], the

110 nature of the plant-specific transporter for phytochelatin or heavy metal

111 glutathione complexes is unknown. Some ABCCs can partially rescue the heavy

112 metal sensitivity of the ycf1 yeast mutant [22], but the substrate transported has

113 not been identified.

114 A member of the AtABCG family, the half-size AtABCG19, confers kanamycin

115 resistance when overexpressed in plants [23]. Since AtABCG19 is highly specific 
116 for kanamycin transport and does not confer resistance to other aminoglycoside

117 antibiotics of clinical importance, the authors propose AtABCG19 as a novel

118 resistance marker for the creation of transgenic plants. Not only would

119 AtABCG19 constitute an endogenous plant-derived marker, it would also limit

120 resistance properties to the antibiotic kanamycin, were it to be transferred

121 horizontally to potentially pathogenic microorganisms.

122 An alternative way for plants to cope with toxic compounds, soilborne heavy

123 metals in particular, is secretion from the cell, aboveground into the apoplast or

124 cuticle and belowground into the rhizosphere. In Arabidopsis, the plasma

125 membrane-intrinsic full-size AtABCG36/AtPDR8, previously described as

126 pathogen defense related (see also chapter 4.), is involved in the detoxification

127 process [7]. Based on the observation that AtABCG36 expression was promoted

128 by exogenously applied cadmium and lead, the authors tested the response of

129 wild type plants, Atabcg36 knock-out mutants, AtABCG36 silenced mutants and

130 AtABCG36 over-expression mutants to elevated heavy metal concentrations. A

131 clear positive correlation between transcript abundance and resistance to lead

132 and cadmium was established. Furthermore, with respect to the wild type, over-

133 expression lines accumulated less cadmium, and silenced lines more cadmium in

134 root and shoot tissues. Direct involvement of AtABCG36 in the export of

135 cadmium ions or cadmium complexes was demonstrated utilizing isolated

136 mesophyll protoplasts in a ${ }^{109} \mathrm{Cadmium}$ flux assay. Whereas an over-expressing

137 line proved more efficient in extruding ${ }^{109} \mathrm{Cadmium}$ than the wildtype, a silenced 
138 line was impaired in its export capacities. The form in which the toxic heavy metal

139 cadmium is transported, whether as free ion or in a chelated form, is unknown.

\section{3. Growth and Development}

\section{3.1. Phytate Transport}

144 Inositol hexakisphosphate (Ins $\mathrm{P}_{6}$, phytate) constitutes a major phosphorus store

145 in plants. It accumulates predominantly in seeds as a complex with magnesium,

146 potassium, calcium, iron and/or zinc, representing up to $5 \%$ of the seed dry-

147 weight [24,25]. During germination phytate is degraded by phytases, thus

148 providing phosphate, myo-inositol and minerals to developing seedlings. Recent

149 publications revealed that InsP $_{6}$ fulfils additional physiological roles. Plants

150 lacking Ins $\mathrm{P}_{6}$ are more susceptible to pathogen attack [26], while in guard cells

151 Ins $\mathrm{P}_{6}$ mobilizes calcium from endomembrane stores and inhibits the inward

152 rectifying $\mathrm{K}^{+}$conductance, thereby influencing stomatal movements $[27,28]$. Ins $\mathrm{P}_{6}$

153 can have a negative impact on the environment, since monogastric animals that

154 lack phytases in their digestive tract fail to process the phytates present in seeds.

155 As a consequence, high amounts of undigested phytates are released with the

156 animal waste into nature, thus accentuating the phosphorus pollution from

157 agriculture [29].

158 During the last few years, low phytate mutants were described in major crop 159 species, i.e. maize, rice and sorghum [30-33]. While in most cases a $45-90 \%$ 160 reduction in seed phytate content did not result in detectable phenotypes, some 
161 of the rice mutant lines proved nonviable. In maize, detailed genetic analysis led

162 to the identification of the ABCC-type transporter ZmMRP4 as the responsible 163 gene behind Ins $\mathrm{P}_{6}$ accumulation in kernels [30]. Phylogenetic studies identified

164 the tonoplastic AtABCC5/AtMRP5 as the closest Arabidopsis homologue to

165 ZmMRP4. Atabcc5 loss-of-function mutants display a low InsP 6 phenotype in

166 seed tissue, which is associated with alterations of mineral cation and phosphate

167 status [34]. Heterologous expression in yeast demonstrated that AtABCC5

168 encodes a specific high affinity Ins $_{6}$ transporter. Moreover, complementation of

169 the Atabcc5 knock-out mutants with an AtABCC5 construct driven by a guard

170 cell-specific promoter restored the sensitivity of the mutant to ABA-mediated

171 inhibition of stomatal opening, supporting the hypothesis that Ins $\mathrm{P}_{6}$ acts as a

172 signaling molecule in guard cells. Hence, it is proposed that by transporting Ins $\mathrm{P}_{6}$

173 into the vacuole, AtABCC5 is essential for the regulation of correct stomatal

174 movement as well as for phytate storage in seeds. One could anticipate that the

175 elucidation of the $\operatorname{Ins}_{6}$ transport mechanism will contribute to the understanding

176 of the regulation of various cellular signalling processes. From an applied

177 perspective it could furthermore address nutritional and environmental questions

178 regarding the possibility of enhancing the quality of seeds by altering the $\operatorname{lns} \mathrm{P}_{6}$

179 content.

\section{$181 \quad 3.2$. Import of fatty acids into peroxisomes}

182 Beta-oxidation of fatty acids is a very important catabolic process, required to

183 generate Acetyl-CoA for entry into the citric acid cycle. In plants it occurs 
184 predominantly within the peroxisomes and thus it requires that fatty acid-CoAs

185 are imported from the cytosol. Arabidopsis loss-of-function mutants of AtABCD1,

186 a full-length peroxisomal $\mathrm{ABC}$ transporter, are strongly impaired in germination

187 and display a pleiotropic growth phenotype. These mutants furthermore exhibit

188 an impaired fatty acid metabolism, suggesting that AtABCD1 is the fatty acid-CoA

189 importer in plant peroxisomes [35-37].

190

\section{3.3. Hormone Transport}

192 Plant development and adaptation to the environment are closely linked to the 193 action of phytohormones. Trytophan derived indole-3-acetic-acid (auxin) is a 194 central phytohormone, implicated in many developmental processes such as 195 shoot elongation, floral bud development, lateral root growth, phototropism and 196 gravitropism [38]. It is mainly produced in the shoot apical meristem, from where 197 it is distributed throughout the plant via cell to cell transport within the xylem 198 parenchyma [38]. Cellular auxin import can either occur simply via diffusion of its 199 protonated form or is catalyzed by members of the AUX1/LAX family of auxin200 influx carriers [39]. Polar export was thought to be governed solely by the PIN 201 family of auxin transporters [39]. However, a detailed examination of two plasma 202 membrane-intrinsic ABCB transporters, AtABCB1 and AtABCB19, suggested that 203 ABC transporters also contribute to inter-cellular auxin transport [40]. Atabcb1 204 and Atabcb19 knock-out plants exhibited slightly retarded growth patterns, while 205 the corresponding double knock-out was strongly impaired in growth, 206 phenotypically reminiscent of auxin deficiency symptoms. Furthermore, auxin 
207 transport in the double knock-out mutant was reduced by more than $70 \%$.

208 Heterologous expression of AtABCB1 and AtABCB19 in several systems

209 presented direct evidence that both proteins are indeed auxin exporters. Recent

210 studies suggest that the two auxin efflux systems are at least partially

211 interconnected. PIN proteins and $\mathrm{ABC}$ transporters interact to modulate the

212 overall auxin transport activity in a complex fashion $[41,42]$. Furthermore, it was

213 shown that the immunophilin-like protein TWD1 interacts with ABCBs,

214 significantly stimulating auxin transport $[43,44]$. In contrast to most members of

215 the PIN family, AtABCB1 and AtABCB19 are expressed only in specific cell types.

216 This, in addition to functional overlaps with PIN proteins, may explain why auxin

217 specific ABCB mutant phenotypes are restricted to impairments in cell elongation

218 of shoot and root.

219 Two recent publications reported that $A B C$ transporters are involved in the

220 transport of the carotenoid-derived stress hormone ABA. Besides being a

221 germination inhibitor present in dormant seeds, ABA is mainly produced in the

222 vasculature of shoots and roots as a response to hydric stress $[45,46]$. From

223 there it is translocated to foliar tissues, where it induces stomata closure to

224 minimize water loss. Consequently, ABA has to be exported from xylem

225 parenchyma cells, transferred to leaves and imported into guard cells and other

226 cell types to trigger the signaling pathways required for coping with water stress.

227 A half-size transporter in the ABCG family, AtABCG25, is expressed in the 228 vascular parenchyma of root and shoot and was shown to act as an ABA 229 exporter with a high affinity for ABA (apparent $\mathrm{Km}$ of $0.2 \mu \mathrm{M}$ ) in heterologous 
230 transport systems [10]. While germination of the corresponding mutant was more

231 sensitive to the application of exogenous ABA, no stomata-specific phenotype

232 could be observed. However, overexpressing AtABCG25 resulted in plants that

233 transpired less water, indicating enhanced ABA export capacities from producing

234 cells. A full-size transporter, AtABCG40, on the other hand, previously proposed

235 to be implicated in heavy metal resistance [6], was found to contribute to the

236 import of ABA across the stomatal plasma membrane [11]. Stomata of Atabcg40

237 mutant plants close less efficiently when the roots are exposed to ABA and the

238 upregulation of ABA-responsive genes upon ABA treatment is delayed. As a

239 consequence, mutant Atabcg40 plants are more sensitive to drought stress. In

240 heterologous systems, AtABCG40 imports ABA with an apparent $\mathrm{Km}$ of $1 \mu \mathrm{M}$,

241 which is within the expected physiological range. Still, the exact mechanism by

242 which AtABCG40 achieves the import of its substrate has yet to be investigated.

243 Import mechanisms are well known from bacterial ABC transporters, which

244 catalyze the uptake of nutrients [47]. In contrast, no ABC importers have been

245 described in the animal field. In plants several full-size ABC transporters have

246 been proposed to act as importers (see also chapter 3.2.). Similarly to AtABCG40,

247 AtABCB14 resides in the stomatal plasma membrane, where it is suggested to

248 govern over the import of apoplastic malate, thus influencing stomatal

249 movements in response to changing carbon dioxide concentrations [48]. An

250 interesting case is AtABCB4, where evidence was presented that, depending on

251 the auxin concentration in the cytosol or medium, it can act as importer or 
252 exporter [49]. Further studies concerning structural aspects are pending to

253 unravel the details of ABC-mediated import in plants.

\section{3.4. Cuticle Formation}

256 The plant cuticle is primarily composed of cuticular waxes (aliphatic very long 257 chain fatty acid derivatives), embedded in a cutin matrix (mainly glycerol, C16 258 and C18 fatty acids) [50]. It covers the epidermis of aerial organs of land plants, 259 forming a protective layer against desiccation and pathogen entry. Proper cuticle 260 deposition is furthermore necessary for organ development, preventing organ 261 fusion and influencing organ morphology. Two half-size ABCG/WBC-proteins, 262 AtABCG11/WBC11 and AtABCG12/WBC12 of Arabidopsis, were demonstrated 263 to play a dominant role in cuticle formation [51,52].

264 AtABCG12, discovered in a forward screen for wax lacking mutants, is expressed 265 in the epidermal tissues of most plant organs and localizes to the plasma 266 membrane, where it is proposed to play a direct role in the export of a multitude 267 of wax precursors [51]. Stem epidermal cells of Atabcg12 knockout plants feature 268 laminar cytoplasmic inclusions of lipidic nature and the stem cuticle is depleted in 269 a variety of wax components such as long chain alkanes, ketones and alcohols, 270 giving the stem a glossy bright green appearance. In contrast, the total amount of 271 epidermal wax, intracellular and cuticular, does not differ between WT and 272 mutant, suggesting that the cytosolic inclusions comprise an accumulation of wax 273 components that are lacking in the cuticle due to transport defects. 
274 AtABCG11 transcript is predominantly found in the epidermis of aerial organs.

275 The protein localizes to the plasma membrane, with a marked polar distribution

276 on the distal epidermal side in developing embryos [53]. In congruence with

277 AtABCG12 function, it contributes to the secretion of cuticular waxes and loss-of-

278 function results in an aberrant cuticle structure [52]. In contrast to Atabcg12,

279 Atabcg11 also displays a reduced cutin load, suggesting cutin precursor fatty

280 acids as additional substrates. The cuticle is one of the main barriers against

281 non-stomatal water loss. Thus, it was intriguing to find that the water stress

282 related hormone $A B A$ induces upregulation of $A t A B C G 11$ [54], which is

283 speculated to enhance fortification of aerial organs against desiccation.

284 Consistent with this finding, Atabcg11 mutants are prone to wilting, but more

285 conspicuously, they display a pleiotropic growth phenotype, with a marked

286 reduction in growth, fusion of rosette leaves, altered reproductive organs and

287 sterility.

288 Double mutants of AtABCG12 and AtABCG11 do not exhibit an additive 289 phenotype [55]. Consequently it was suggested that they form heterodimers, with 290 a strong affinity for cuticular waxes. The additional cutin phenotypes of Atabc11 291 mutants suggest that AtABCG11 can also form homodimers or heterodimers with 292 half-size ABCG members other than AtABCG12 to create a complex with a high 293 affinity for cutin precursor fatty acids. 
296 Anti-microbial plant secondary metabolites such as terpenoid derivates and

297 cyanogenic glycosides form an important first line of defense against host and 298 non-host pathogens [56]. They inhibit the proliferation of fungal and bacterial 299 microbes on aerial plant surfaces, within the rhizosphere and in the apoplast 300 around local infection sites. There is increasing evidence that the aboveground 301 and belowground secretion of such compounds is in part mediated by full-size 302 ABC transporters of the ABCG/PDR subfamily. Transcript profiling of $A B C G$ 303 members in rice [57] revealed that nearly half of them positively respond to 304 jasmonic acid (JA) and/or salicylic acid (SA), two phytohormones implicated in 305 biotic stress responses. This suggests roles in pathogen defense, which is 306 supported by the finding that three full-length $A B C G$ s are induced in rice leaves 307 that are infected with the biotrophic fungus Magnaporthe grisea. Belowground, 308 loss-of-function of AtABCG30/AtPDR2 results in a drastic change of soil 309 microflora in the rhizosphere [58], which the authors attribute to a change in root310 exudate composition. Additionally, the overall profile of root exudates is altered in 311 several ABC transporter mutants [59].

312 Particularly within the rhizosphere, plant-derived secondary compounds serve as 313 powerful attractants for beneficial microbes such as mycorrhizal fungi and 314 rhizobacteria. Specific inhibitor studies suggest that ABCG subfamily members 315 are responsible for the secretion of genistein and daidzein, both iso-flavonoids 316 that act as plant-derived signaling molecules in the legume-rhizobia symbiosis, 317 into the rhizosphere $[60,61]$. 
318 Functional characterization of NpPDR1, a full-size ABCG protein of Nicotiana

319 plumbaginifolia, was the first report of a plant $A B C$ transporter being implicated in

320 pathogen defense and the first description of active terpenoid transport in plants

321 [62]. NpPDR1 resides in the plasma membrane and is induced by the anti-fungal

322 diterpenes sclareol and sclareolide. In isolated microsomes NpPDR1 contributes

323 to the transport of radiolabeled compounds closely related to sclareol, supporting

324 the hypothesis that sclareol is a natural substrate. NpPDR1 transcript is most

325 abundant in the leaf epidermis, including leaf trichomes, but is also present in

326 root tissues and petals [13]. Apart from its proposed substrate sclareol, both JA

327 and SA promote NpPDR1 expression, indicating association with defense-related

328 signaling pathways. Concomitantly, a strong response to Botritis cinerea and

329 Pseudomonas syringae, both necrotrophic non-host pathogens, is observed.

330 Downregulation of NpPDR1 lead to spontaneous and commonly lethal infections

331 with $B$. cinerea and rendered the plant highly susceptible to exogenously applied

332 sclareol. All these findings indicate a participation of NpPDR1 in basal plant

333 defense. It is hypothesized that antimicrobial compounds such as sclareol are

334 deposited via NpPDR1 on the leaf surface and possibly exuded into the 335 rhizosphere, contributing to a constitutive chemical defense barrier. Upon

336 perception of various pathogens and transduction via JA and SA dependent 337 pathways this mode of defense is intensified locally around areas of infection, so

338 that NpPDR1-mediated modes of action are both constitutive and induced.

339 AtABCG36/AtPDR8/PEN3 was first recognized as a crucial factor in pre-invasive 340 nonhost resistance in an extensive forward genetic screen of Arabidopsis 
341 mutants for an increased susceptibility to the barley powdery mildew pathogen

342 [12]. The AtABCG36 loss-of-function mutants were compromised in their capacity

343 to prevent entry of two nonhost biotrophs and one nonhost necrotroph. However,

344 they proved hyperresistant against the compatible Arabidopsis powdery mildew

345 pathogen, which the authors attribute to a hyperactivation of SA-dependent

346 pathways observed in the mutant. GFP-fusion construct under the control of the

347 native promoter complemented the phenotype and the corresponding fusion

348 protein was targeted to the plasma membrane, with increased fluorescence

349 intensities at infection sites. The authors hypothesize that AtABCG36 transports

350 defense related compounds across the plasma membrane in a concentrated

351 manner at local infection sites. Lack of AtABCG36 function would lead to an

352 accumulation of these compounds within the cell, which may activate SA

353 dependent pathways that prime defense against compatible pathogens.

354 Recently a full-size $A B C G$ was identified as the responsible gene behind a robust

355 and durable pathogen resistance against leaf rust, stripe rust and powdery

356 mildew in wheat carrying functional LR34 (Leaf Rust 34) alleles [63]. Unlike many

357 other ABCGs, its expression seems to be modulated by developmental cues

358 rather than stress factors. LR34 is predominantly found in adult foliar tissues,

359 particularly the flag leaf. Transcript abundance is highest in the leaf tip and wheat

360 varieties with functional $L R 34$ alleles can be phenotypically selected via a leaf tip

361 necrosis developing in adult flag leaves. Despite its resistance conferring

362 properties, LR34 is not responsive to pathogen inoculation, suggesting rather

363 constitutive than induced functions. In contrast to NpPDR1 and AtABCG36 
364 function, which seem restricted to nonhost resistance, LR34 is implicated in the

365 defense against several compatible pathogens of fungal origin. LR34 is the only

366 ABCG protein characterized to date, that impedes the invasion and spread of

367 compatible pathogens. Research with focus on the nature of its substrates is

368 ongoing.

369 Considering the participation of ABCG transporters in secondary metabolite-

370 based pathogen response, it is reasonable to assume that they also play a role in

371 herbivory defense, e.g. via the deposition of insect-deterring compounds on leaf

372 surfaces. Certain compounds of terpenoid origin are known to act as herbivore

373 deterrents and JA, a potent inducer of many full-size ABCGs, is also a major

374 mediator of herbivory responses.

375

376 5. Conclusion

377 The functional study of plant $A B C$ transporters is a rapidly expanding field. It is

378 becoming increasingly apparent that $A B C$ dependent transport processes not

379 only serve to protect the plant from endogenous and exogenous toxic

380 compounds, but that they are indispensable for proper development, adequate

381 interaction with the environment and basic metabolic processes.

382 There seems to be no clear functional separation among the different subfamilies,

383 but from our current knowledge it is possible to define subfamily specific

384 preferences. Members of the ABCB subfamily have been mainly implicated in

385 organic acid transport across the plasma membrane and it is probable that other

386 members serve similar functions, e.g. in the excretion of carboxylates for either 
387 cytosolic $\mathrm{pH}$ homeostasis or rhizosphere acidification. Secondary compounds of 388 terpenoid origin and highly lipophilic compounds are common substrates for 389 members of the ABCG clade. The question remains whether ABCG proteins 390 might also display specificity for other secondary compound classes such as

391 alkaloids and flavonoids. Biotechnological approaches to produce natural 392 compounds of medicinal value in heterologous or modified tissue culture systems 393 are currently pursued with emphasis to supply the pharmaceutical market. 394 Understanding and designing highly productive systems must necessarily include 395 efficient transport mechanisms to relocate precursors within the manufacturing 396 units and to excrete final products into the medium. The ability of several 397 subfamily members to efficiently sequester xenobiotic compounds and heavy 398 metals is also of importance to the biotechnological market. Attempts to detoxify 399 contaminated soils via phytoremediation, utilizing genetically modified plants, are 400 underway. Altered expression of certain $A B C$ transporters appears to be part of a 401 feasible approach, for example, to prevent reflux of extracted contaminants into 402 the soil and enhance vacuolar accumulation in foliar tissues.

403 The novel findings that $A B C G$ members are participating in $A B A$ transport are 404 intriguing and tempt us to speculate that the translocation of hormones other than $405 \mathrm{ABA}$ and auxin might also rely on $\mathrm{ABC}$-dependent mechanisms. Possible 406 candidates falling into the predicted substrate range of ABCGs are the recently 407 discovered branching hormone strigolactone [64,65], an apocarotenoid 408 structurally related to ABA, and the brassinosteroids, highly lipophilic terpenoid 409 derivates. 
$410 \mathrm{ABC}$ proteins play an important role in plant growth and development. The

411 function of most of plant $A B C$ proteins still awaits elucidation. Functional

412 redundancies render classical mutagenesis-based forward- or reverse-genetic

413 approaches futile. Producing multiple loss-of-function mutants of closely related

$414 \mathrm{ABC}$ transporters, and/or finding their interacting partners might reveal additional

415 functions that will direct the studies on plant $A B C$ transporters.

416

417 6. Summary

418 - Higher plants contain more than one hundred ABC proteins that are 419 organized in eight clusters, namely $A B C A$ to $A B C l$ subfamilies, and 420 predominantly localize to the plasma membrane and tonoplast.

- Most ABCCs/MRPs localize to the tonoplast where several of them are thought to catalyze the vacuolar sequestration of xenobiotic-conjugates.

- AtABCG36 plays a role in heavy metal tolerance, as well as in pathogen defense, but the nature of the respective substrates has yet to be

- AtABCC5 is a tonoplastic phytate transporter with implications in phytate storage in seeds and regulation of stomatal movement. 
- Several ABCBs/PGPs are participating in a directional cell to cell transport of auxin via polar export and interaction with other auxin transporters.

- Two ABCGs contribute to the inter-cellular transport of the apocarotenoid stress hormone ABA.

- Two half-size ABCGs/WBCs contribute to cuticle formation via the epidermal excretion of cuticular wax components.

- Several full-size ABCGs/PDRs have been implicated in pathogen defense supposedly via excretion of anti-microbial secondary metabolites.

- AtABCB14 localizes to the plasma membrane of guard cells and catalyzes the import of malate, thus influencing stomatal movement.

\section{References}

1. Verrier PJ, Bird D, Burla B, Dassa E, Forestier C, Geisler M, Klein M, Kolukisaoglu Ü, Lee Y, Martinoia E, et al. (2008) Plant ABC proteins - a unified nomenclature and updated inventory. Trends in Plant Science 13,151-159.

2. Rea PA (2007) Plant ATP-binding cassette transporters. Annu Rev Plant Biol 58,347-375.

3. Mason DL, Michaelis S (2002) Requirement of the N-Terminal Extension for Vacuolar Trafficking and Transport Activity of Yeast Ycf1p, an ATP-binding Cassette Transporter

10.1091/mbc.E02-07-0405. Mol. Biol. Cell 13,4443-4455.

4. Westlake CJ, Cole SPC, Deeley RG (2005) Role of the NH2-terminal membrane spanning domain of multidrug resistance protein 1/ABCC1 in protein processing and trafficking. Mol Biol Cell 16,2483-2492. 
5. Yazaki K, Shitan N, Sugiyama A, Takanashi K: Chapter 6 Cell and Molecular Biology of ATP-Binding Cassette Proteins in Plants. International Review of Cell and Molecular Biology. edn Volume 276. Edited by Jeon KW: Academic Press; 2009:263-299.

6. Lee M, Lee K, Lee J, Noh EW, Lee Y (2005) AtPDR12 contributes to lead resistance in Arabidopsis. Plant Physiol 138,827-836.

7. Kim D-Y, Bovet L, Maeshima M, Martinoia E, Lee Y (2007) The ABC transporter AtPDR8 is a cadmium extrusion pump conferring heavy metal resistance. Plant J 50,207-218.

8. Klein M, Burla B, Martinoia E (2006) The multidrug resistance-associated protein (MRP/ABCC) subfamily of ATP-binding cassette transporters in plants. FEBS Lett 580,1112-1122.

9. Geisler M, Murphy AS (2006) The ABC of auxin transport: the role of $p$ glycoproteins in plant development. FEBS Lett 580,1094-1102.

10. Kuromori T, Miyaji T, Yabuuchi $H$, Shimizu $H$, Sugimoto E, Kamiya A, Moriyama Y, Shinozaki K (2010) ABC transporter AtABCG25 is involved in abscisic acid transport and responses. Proc Natl Acad Sci U S A 107,2361-2366.

11. Kang J, Hwang J-U, Lee M, Kim Y-Y, Assmann SM, Martinoia E, Lee Y (2010) PDR-type ABC transporter mediates cellular uptake of the phytohormone abscisic acid. Proc Natl Acad Sci U S A 107,2355-2360.

12. Stein M, Dittgen J, Sanchez-Rodriguez C, Hou B-H, Molina A, Schulze-Lefert P, Lipka V, Somerville S (2006) Arabidopsis PEN3/PDR8, an ATP binding cassette transporter, contributes to nonhost resistance to inappropriate pathogens that enter by direct penetration. Plant Cell 18,731-746.

13. Stukkens Y, Bultreys A, Grec S, Trombik T, Vanham D, Boutry M (2005) NpPDR1, a pleiotropic drug resistance-type ATP-binding cassette transporter from Nicotiana plumbaginifolia, plays a major role in plant pathogen defense. Plant Physiol 139,341-352.

14. Martinoia E, Grill E, Tommasini R, Kreuz K, Amrhein N (1993) ATPdependent glutathione S-conjugate 'export' pump in the vacuolar membrane of plants. 364,247-249.

15. Kreuz K, Tommasini R, Martinoia E (1996) Old Enzymes for a New Job (Herbicide Detoxification in Plants). Plant Physiol 111,349-353.

16. Lu YP, Li ZS, Drozdowicz YM, Hortensteiner S, Martinoia E, Rea PA (1998) AtMRP2, an Arabidopsis ATP binding cassette transporter able to transport glutathione S-conjugates and chlorophyll catabolites: functional comparisons with Atmrp1. Plant Cell 10,267-282.

17. Raichaudhuri A, Peng M, Naponelli V, Chen S, Sanchez-Fernandez R, Gu H, Gregory JF, Hanson AD, Rea PA (2009) Plant Vacuolar ATP-binding Cassette Transporters That Translocate Folates and Antifolates in Vitro and Contribute to Antifolate Tolerance in Vivo. J Biol Chem 284,84498460.

18. Jaquinod M, Villiers F, Kieffer-Jaquinod S, Hugouvieux V, Bruley C, Garin J, Bourguignon J (2007) A Proteomics Dissection of Arabidopsis thaliana Vacuoles Isolated from Cell Culture. Mol Cell Proteomics 6,394-412. 
19. Salt DE, Rauser WE (1995) MgATP-Dependent Transport of Phytochelatins Across the Tonoplast of Oat Roots. Plant Physiol 107,1293-1301.

20. Szczypka MS, Wemmie JA, Moye-Rowley WS, Thiele DJ (1994) A yeast metal resistance protein similar to human cystic fibrosis transmembrane conductance regulator (CFTR) and multidrug resistance-associated protein. J Biol Chem 269,22853-22857.

21. Ortiz DF, Kreppel L, Speiser DM, Scheel G, McDonald G, Ow DW (1992) Heavy metal tolerance in the fission yeast requires an ATP-binding cassette-type vacuolar membrane transporter. EMBO J 11,3491-3499.

22. Tommasini R, Vogt E, Fromenteau M, Hortensteiner S, Matile P, Amrhein N, Martinoia E (1998) An ABC-transporter of Arabidopsis thaliana has both glutathione-conjugate and chlorophyll catabolite transport activity. Plant $\mathrm{J}$ 13,773-780.

23. Mentewab A, Stewart CN (2005) Overexpression of an Arabidopsis thaliana $\mathrm{ABC}$ transporter confers kanamycin resistance to transgenic plants. Nat Biotechnol 23,1177-1180.

24. Raboy V (2007) The ABCs of low-phytate crops. Nat Biotechnol 25,874-875.

25. Raboy V (2003) myo-Inositol-1,2,3,4,5,6-hexakisphosphate. Phytochemistry 64,1033-1043.

26. Murphy AM, Otto B, Brearley CA, Carr JP, Hanke DE (2008) A role for inositol hexakisphosphate in the maintenance of basal resistance to plant pathogens. Plant J 56,638-652.

27. Lemtiri-Chlieh F, MacRobbie EA, Brearley CA (2000) Inositol hexakisphosphate is a physiological signal regulating the $\mathrm{K}_{+}$-inward rectifying conductance in guard cells. Proc Natl Acad Sci U S A 97,86878692.

28. Lemtiri-Chlieh F, MacRobbie EAC, Webb AAR, Manison NF, Brownlee C, Skepper JN, Chen J, Prestwich GD, Brearley CA (2003) Inositol hexakisphosphate mobilizes an endomembrane store of calcium in guard cells. Proc Natl Acad Sci U S A 100,10091-10095.

29. Coffey GCaR: Phosphorus-a key essential nutrient, yet a possible major pollutant-its central role in animal nutrition. In Lyons, T. P. eds. Biotechnology in the Feed Industry. Edited by; 1991:133-145.

30. Shi J, Wang H, Schellin K, Li B, Faller M, Stoop JM, Meeley RB, Ertl DS, Ranch JP, Glassman K (2007) Embryo-specific silencing of a transporter reduces phytic acid content of maize and soybean seeds. Nat Biotechnol 25,930-937.

31. Shi J, Wang H, Wu Y, Hazebroek J, Meeley RB, Ertl DS (2003) The maize low-phytic acid mutant lpa2 is caused by mutation in an inositol phosphate kinase gene. Plant Physiol 131,507-515.

32. Rasmussen SK, Hatzack F (1998) Identification of two Low-Phytate Barley (Hordeum Vulgare) Grain Mutants by TLC and Genetic Analysis. Hereditas 129,107-112.

33. Xu X-H, Zhao H-J, Liu Q-L, Frank T, Engel K-H, An G, Shu Q-Y (2009) Mutations of the multi-drug resistance-associated protein $A B C$ transporter 
562

563

564

565

566

567

568

586

587

588

589

590

591

592

593

594

595

596 gene 5 result in reduction of phytic acid in rice seeds. Theor Appl Genet 119,75-83.

34. Nagy R, Grob H, Weder B, Green $P$, Klein M, Frelet-Barrand A, Schjoerring JK, Brearley C, Martinoia E (2009) The Arabidopsis ATP-binding cassette protein AtMRP5/AtABCC5 is a high affinity inositol hexakisphosphate transporter involved in guard cell signaling and phytate storage. J Biol Chem 284,33614-33622.

35. Footitt S, Slocombe SP, Larner V, Kurup S, Wu Y, Larson T, Graham I, Baker A, Holdsworth M (2002) Control of germination and lipid mobilization by COMATOSE, the Arabidopsis homologue of human ALDP. EMBO J 21,2912-2922.

36. Hayashi M, Nito K, Takei-Hoshi R, Yagi M, Kondo M, Suenaga A, Yamaya T, Nishimura M (2002) Ped3p is a peroxisomal ATP-binding cassette transporter that might supply substrates for fatty acid beta-oxidation. Plant Cell Physiol 43,1-11.

37. Zolman BK, Silva ID, Bartel B (2001) The Arabidopsis pxa1 mutant is defective in an ATP-binding cassette transporter-like protein required for peroxisomal fatty acid beta-oxidation. Plant Physiol 127,1266-1278.

38. Vanneste S, Friml J (2009) Auxin: a trigger for change in plant development. Cell 136,1005-1016.

39. Kramer EM (2004) PIN and AUX/LAX proteins: their role in auxin accumulation. Trends in Plant Science 9,578-582.

40. Geisler M, Blakeslee JJ, Bouchard R, Lee OR, Vincenzetti V, Bandyopadhyay A, Titapiwatanakun B, Peer WA, Bailly A, Richards EL, et al. (2005) Cellular efflux of auxin catalyzed by the Arabidopsis MDR/PGP transporter AtPGP1. Plant J 44,179-194.

41. Bandyopadhyay A, Blakeslee JJ, Lee OR, Mravec J, Sauer M, Titapiwatanakun B, Makam SN, Bouchard R, Geisler M, Martinoia E, et al. (2007) Interactions of PIN and PGP auxin transport mechanisms. Biochem Soc Trans 35,137-141.

42. Blakeslee JJ, Bandyopadhyay A, Lee OR, Mravec J, Titapiwatanakun B, Sauer M, Makam SN, Cheng Y, Bouchard R, Adamec J, et al. (2007) Interactions among PIN-FORMED and P-glycoprotein auxin transporters in Arabidopsis. Plant Cell 19,131-147.

43. Bailly A, Sovero V, Vincenzetti V, Santelia D, Bartnik D, Koenig BW, Mancuso S, Martinoia E, Geisler M (2008) Modulation of P-glycoproteins by auxin transport inhibitors is mediated by interaction with immunophilins. J Biol Chem 283,21817-21826.

44. Geisler M, Kolukisaoglu HU, Bouchard R, Billion K, Berger J, Saal B, Frangne N, Koncz-Kalman Z, Koncz C, Dudler R, et al. (2003) TWISTED DWARF1, a unique plasma membrane-anchored immunophilin-like protein, interacts with Arabidopsis multidrug resistance-like transporters AtPGP1 and AtPGP19. Mol Biol Cell 14,4238-4249.

45. Finkelstein R, Reeves W, Ariizumi T, Steber C (2008) Molecular Aspects of Seed Dormancy*. Annu. Rev. Plant Biol. 59,387-415. 
608

609

610

611

612

613

614

615

46. Schachtman DP, Goodger JQD (2008) Chemical root to shoot signaling under drought. Trends in Plant Science 13,281-287.

47. Davidson AL, Dassa E, Orelle C, Chen J (2008) Structure, function, and evolution of bacterial ATP-binding cassette systems. Microbiol Mol Biol Rev 72,317-364.

48. Lee M, Choi Y, Burla B, Kim Y-Y, Jeon B, Maeshima M, Yoo J-Y, Martinoia E, Lee $Y(2008)$ The $A B C$ transporter AtABCB14 is a malate importer and modulates stomatal response to CO2. Nat Cell Biol 10,1217-1223.

49. Yang $\mathrm{H}$, Murphy AS (2009) Functional expression and characterization of Arabidopsis ABCB, AUX 1 and PIN auxin transporters in Schizosaccharomyces pombe. Plant J 59,179-191.

50. Samuels L, Kunst L, Jetter R (2008) Sealing plant surfaces: cuticular wax formation by epidermal cells. Annu Rev Plant Biol 59,683-707.

51. Pighin JA, Zheng $H$, Balakshin LJ, Goodman IP, Western TL, Jetter R, Kunst L, Samuels AL (2004) Plant cuticular lipid export requires an ABC transporter. Science 306,702-704.

52. Panikashvili D, Savaldi-Goldstein S, Mandel T, Yifhar T, Franke RB, Hofer R, Schreiber L, Chory J, Aharoni A (2007) The Arabidopsis

DESPERADO/AtWBC11 transporter is required for cutin and wax secretion. Plant Physiol 145,1345-1360.

53. Panikashvili D, Shi JX, Bocobza S, Franke RB, Schreiber L, Aharoni A (2009) The Arabidopsis DSO/ABCG11 Transporter Affects Cutin Metabolism in Reproductive Organs and Suberin in Roots. Mol Plant.

54. Luo B, Xue X-Y, Hu W-L, Wang L-J, Chen X-Y (2007) An ABC transporter gene of Arabidopsis thaliana, AtWBC11, is involved in cuticle development and prevention of organ fusion. Plant Cell Physiol 48,1790-1802.

55. Bird D, Beisson F, Brigham A, Shin J, Greer S, Jetter R, Kunst L, Wu X, Yephremov A, Samuels L (2007) Characterization of Arabidopsis ABCG11/WBC11, an ATP binding cassette (ABC) transporter that is required for cuticular lipid secretion. Plant J 52,485-498.

56. Osbourn AE (1996) Preformed Antimicrobial Compounds and Plant Defense against Fungal Attack. Plant Cell 8,1821-1831.

57. Moons A (2008) Transcriptional profiling of the PDR gene family in rice roots in response to plant growth regulators, redox perturbations and weak organic acid stresses. Planta 229,53-71.

58. Badri DV, Quintana N, El Kassis EG, Kim HK, Choi YH, Sugiyama A, Verpoorte R, Martinoia E, Manter DK, Vivanco JM (2009) An ABC transporter mutation alters root exudation of phytochemicals that provoke an overhaul of natural soil microbiota. Plant Physiol 151,2006-2017.

59. Badri DV, Loyola-Vargas VM, Broeckling CD, De-la-Pena C, Jasinski M, Santelia D, Martinoia E, Sumner LW, Banta LM, Stermitz F, et al. (2008) Altered profile of secondary metabolites in the root exudates of Arabidopsis ATP-binding cassette transporter mutants. Plant Physiol 146,762-771. 

671 tonoplast and mediate the transport of xenobiotic compounds and endogenous

672 metabolites into the vacuole. AtABCG11/12 are polarly localized on the plasma

673 membrane and export lipids used for cuticle formation. AtABCG36 is a resistance rhizobium: An ATP-binding cassette-type transporter mediates genistein secretion. Plant Signal Behav 3,38-40.

61. Sugiyama A, Shitan N, Yazaki K (2007) Involvement of a soybean ATPbinding cassette-type transporter in the secretion of genistein, a signal flavonoid in legume-Rhizobium symbiosis. Plant Physiol 144,2000-2008.

62. Jasinski M, Stukkens Y, Degand H, Purnelle B, Marchand-Brynaert J, Boutry M (2001) A plant plasma membrane ATP binding cassette-type transporter is involved in antifungal terpenoid secretion. Plant Cell 13,1095-1107.

63. Krattinger SG, Lagudah ES, Spielmeyer W, Singh RP, Huerta-Espino J, McFadden H, Bossolini E, Selter LL, Keller B (2009) A Putative ABC Transporter Confers Durable Resistance to Multiple Fungal Pathogens in Wheat. Science.

64. Umehara M, Hanada A, Yoshida S, Akiyama K, Arite T, Takeda-Kamiya N, Magome H, Kamiya Y, Shirasu K, Yoneyama K, et al. (2008) Inhibition of shoot branching by new terpenoid plant hormones. Nature 455,195-200.

65. Gomez-Roldan V, Fermas S, Brewer PB, Puech-Pages V, Dun EA, Pillot J-P, Letisse F, Matusova R, Danoun S, Portais J-C, et al. (2008) Strigolactone inhibition of shoot branching. Nature 455,189-194.

\section{Figure legends}

Figure 1. Arabidopsis $A B C$ transporters in different organs with their substrate and/or function.

Figure 2. Tissue level localization of Arabidopsis $A B C$ transporters with their substrates

(a) Mesophyll and epidermal cells. AtABCC1 and AtABCC2 reside on the 
674 factor for pathogens. (b) Guard cell. AtABCG40 acts as an ABA importer from the 675 apoplast into the cytosol and mediates ABA induced stomatal closure. The 676 tonoplast localized AtABCC5 transports $\operatorname{InsP}_{6}\left(\mathrm{IP}_{6}\right)$ into the vacuole thereby 677 lowering the active pool of InsP $_{6}$ in the cytosol. AtABCB14 is a plasma678 membrane malate importer that acts during stomatal movements. The major 679 outward current of malate during stomatal closure is indicated with a gapped blue 680 arrow. (c) Transection through a root. AtABCG25 is polarly localized on the 681 plasma membrane of parenchyma cells where it mediates $A B A$ export from 682 parenchyma cells into the xylem stream. AtABCG36 has been shown to export 683 toxic cadmium ions that have unspecifically entered epidermal cells. AtABCG30 684 is an exporter for root exudates and resides on the plasma membrane of root 685 epidermal cells.

686 


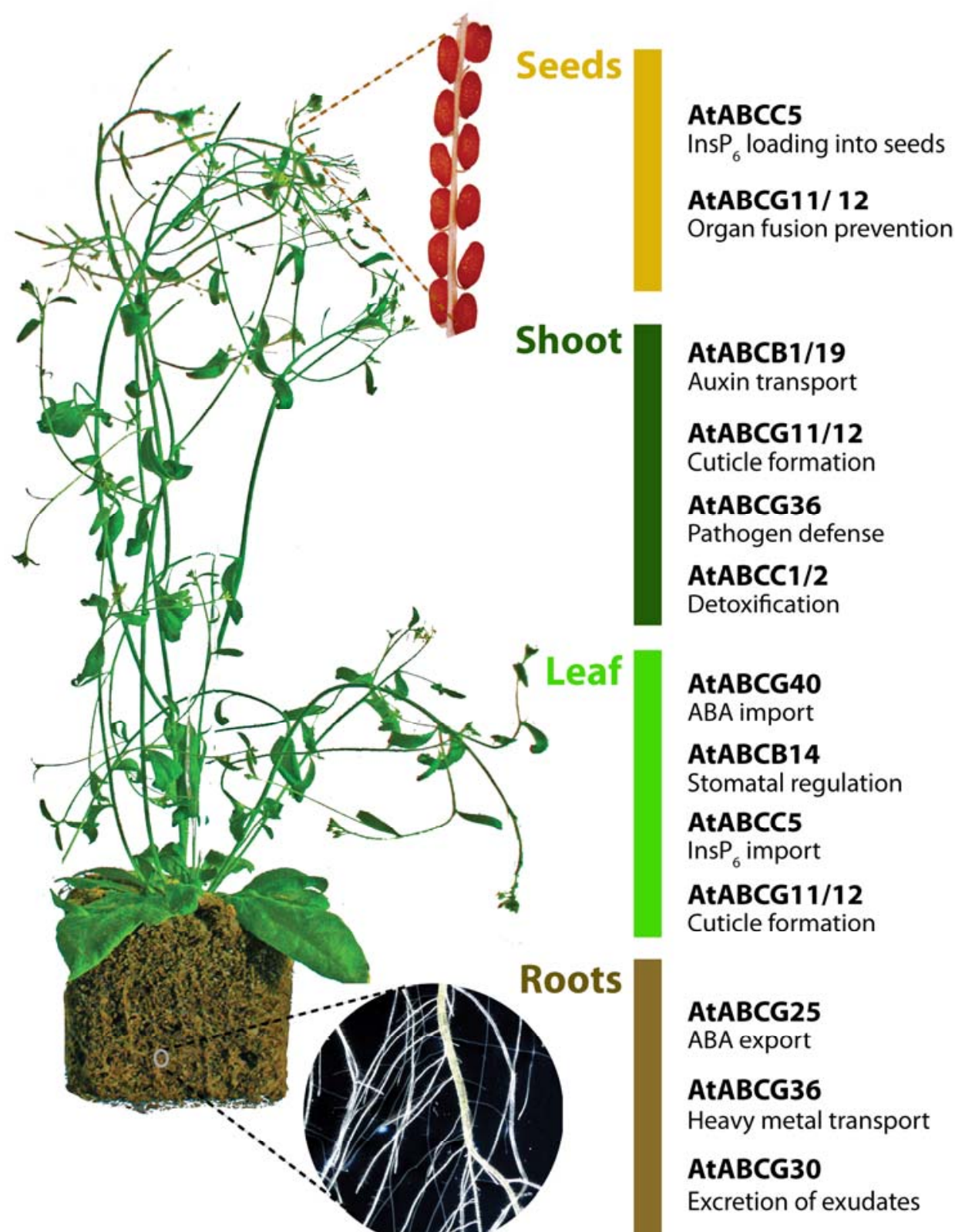

690 
10. Figure 2
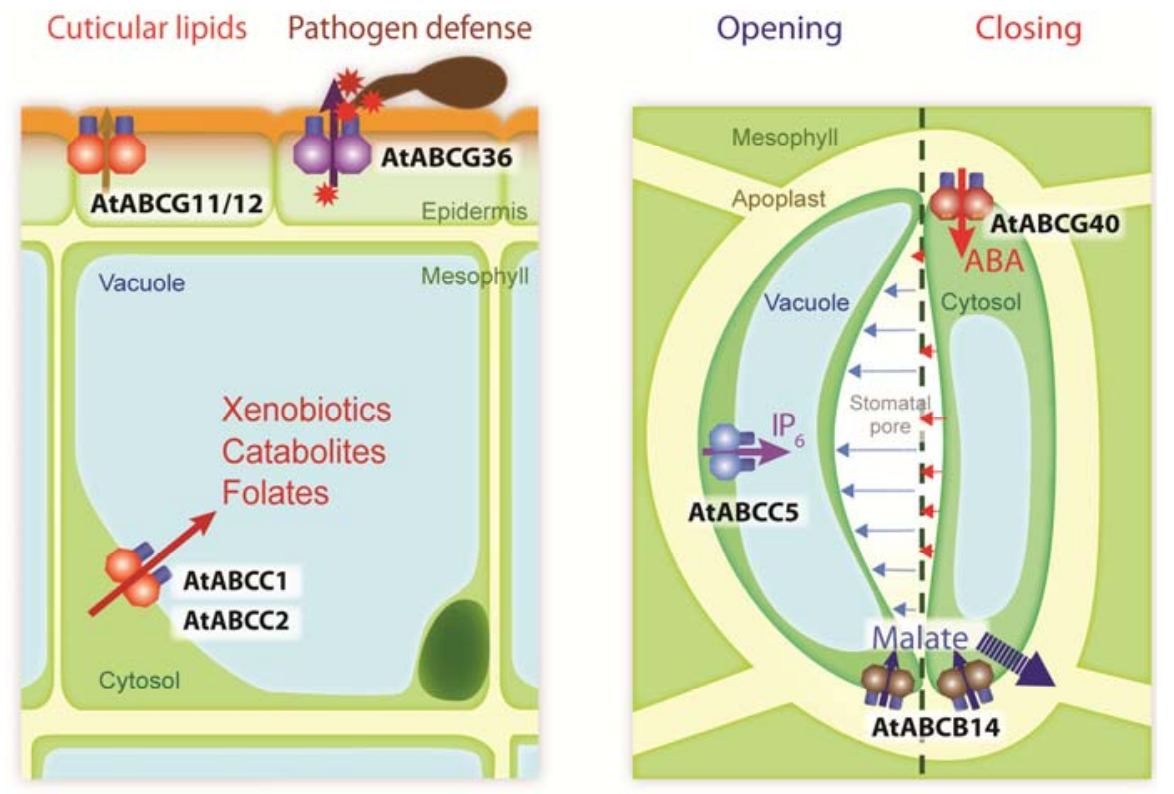

696

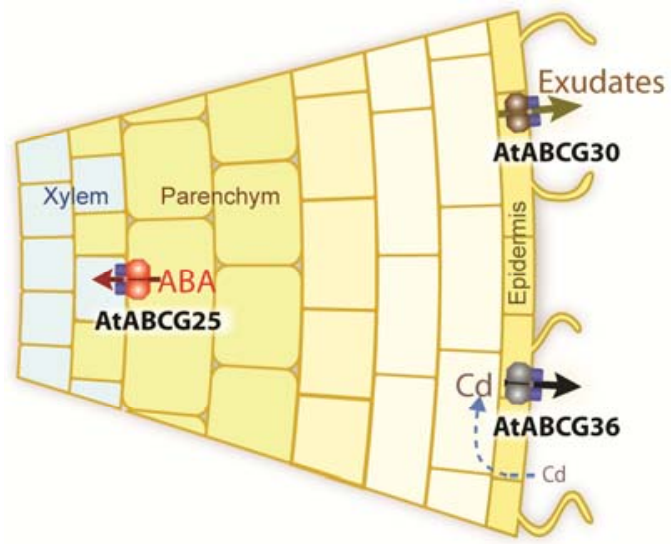

697

698

699

700

701

702

10. Key words

703 
704 Arabidopsis thaliana

705 ABCB/PGP subfamily

706 ABCC/MRP subfamily

707 Half-size ABCG/WBC subfamily

708 Full-size ABCG/PDR subfamily

709 Export and Import

710 Cellular detoxification

711 Phytate transport

712 Hormone transport

713 Stomatal movements,

714 Cuticle formation

715 Pathogen defense

716 Transport of natural compounds 